\title{
Bounding the distance among longest paths in a connected graph
}

\author{
Jan Ekstein* Shinya Fujita ${ }^{\dagger} \quad$ Adam Kabela* Jakub Teska* $^{*}$ \\ May 4, 2018
}

\begin{abstract}
It is easy to see that in a connected graph any 2 longest paths have a vertex in common. For $k \geq 7$, Skupień in 1966 obtained a connected graph in which some $k$ longest paths have no common vertex, but every $k-1$ longest paths have a common vertex. It is not known whether every 3 longest paths in a connected graph have a common vertex and similarly for 4,5, and 6 longest path. Fujita et al. in 2015 give an upper bound on distance among 3 longest paths in a connected graph. In this paper we give a similar upper bound on distance between 4 longest paths and also for $k$ longest paths, in general.
\end{abstract}

\section{Introduction}

In 1966 Gallai in [4] asked whether all longest paths in a connected graph have a vertex in common. Couple of years later, several counterexamples were found, see [9], [10], and [11]. In 1976 Thomassen in [8] showed that there exist infinitely many counterexamples to Gallai's question.

On the other hand, if we restrict to a special class of graphs, the answer to Gallai's question may become positive. For example in a tree, all longest paths must have a vertex in common. Klavžar and Petkovšek in [6] proved that it is also true for split graphs and cacti and Balister et al. in [2] proved it for the class of circular arc graphs.

Another approach to Gallai's question is to ask, what happens if we consider a fixed number of longest paths. It is easy to see that every 2 longest paths in a connected graph have a common vertex. For 3 longest paths, the question remains open. This has been originally asked by Zamfirescu in [12].

*Department of Mathematics, Institute for Theoretical Computer Science, and European Centre of Excellence NTIS - New Technologies for the Information Society, Faculty of Applied Sciences, University of West Bohemia, Pilsen, Technická 8, 30614 Plzeň, Czech Republic, e-mail: \{ekstein, kabela, teska\}@kma.zcu.cz.

†International College of Arts and Sciences, Yokohama City University, 22-2 Seto, Kanazawa-ku, Yokohama 236-0027, Japan, e-mail: shinya.fujita.ph.d@gmail.com. 
Conjecture 1. 12] For every connected graph, any 3 of its longest paths have a common vertex.

There are few results dealing with this conjecture. Axenovich in [1] proved that it is true for connected outerplanar graphs and de Rezende et al. in [3] showed that Conjecture 1 is true for connected graphs in which all nontrivial blocks are hamiltonian.

For $k \geq 7$, Skupień in [7] obtained a connected graph in which some $k$ longest paths have no common vertex, but every $k-1$ longest paths have a common vertex. Regarding this, it is still valid to ask wheter not only 3 but also 4,5 , and 6 longest path in a connected graph have a common vertex.

In [5] the authors introduced a parameter to measure the distance among the longest paths in a connected graph and proved an upper bound of this parameter for 3 longest paths. To state their result we give some definitions first.

Let $G$ be a connected graph. Let $\ell(G)$ be the length of any longest path in $G$ and $\mathcal{L}(G)=\{P \mid P$ is a path in $G$ with $|V(P)|=\ell(G)+1\}$ be a set of longest paths of $G$. For $x, y \in V(G)$, let $d_{G}(x, y)$ be the distance between $x$ and $y$ in $G$. For a vertex $x \in V(G)$ and a subset $U \subseteq V(G)$, let $d_{G}(x, U)=\min \left\{d_{G}(x, y) \mid y \in U\right\}$. For $\mathcal{P} \subseteq \mathcal{L}(G)$ we call path-distance-function $f(G, \mathcal{P})=\min \left\{\sum_{P \in \mathcal{P}} d_{G}(v, V(P)) \mid v \in V(G)\right\}$.

For a class of graphs $\mathcal{G}$ and an integer $k$, we introduce path-distance-ratio $d_{k}(\mathcal{G})=$

$\max \frac{f(G, \mathcal{P})}{|V(G)|}$, where the maximum is taken over all the graphs of $\mathcal{G}$ and their sets of longest paths $\mathcal{P} \subseteq \mathcal{L}(G)$ with $|\mathcal{P}|=k$.

Let $\mathcal{G}_{c}$ be a class of connected graphs. The question whether for every connected graph any 3 longest paths have a vertex in common translates into the question whether $d_{3}\left(\mathcal{G}_{c}\right)=0$. On the other hand, Skupien in [7] constructed a graph on 17 vertices, in which there are 7 longest paths without a common vertex, this graph implies that $d_{7}\left(\mathcal{G}_{c}\right) \geq \frac{1}{17}$.

Now we can state the result by Fujita et al. from [5].

Theorem 2. [5] Let $\mathcal{G}_{c}$ be a class of connected graphs. Then $d_{3}\left(\mathcal{G}_{c}\right) \leq \frac{1}{17}$.

In this paper we prove similar results for 4 longest path and also for $k$ longest paths, in general.

Theorem 3. Let $\mathcal{G}_{c}$ be a class of connected graphs. Then $d_{4}\left(\mathcal{G}_{c}\right) \leq \frac{3}{16}$.

By picking any vertex of a connected graph $G$, we see that $d_{k}\left(\mathcal{G}_{c}\right)$ can be bounded by $k$. We show that it can be improved as roughly $\frac{k}{6}$.

Theorem 4. Let $\mathcal{G}_{c}$ be a class of connected graphs and let $k \geq 3$ be an integer. Then $d_{k}\left(\mathcal{G}_{c}\right) \leq \frac{k^{3}-4 k^{2}+5 k-2}{6 k^{2}-8 k}$.

\section{Proofs}

In our proofs, we adapt ideas of [5]. We start by giving several technical definitions. 
Let $G$ be a connected graph. Let $U$ and $V$ be two sets of vertices of $G$, let $P$ be a path in $G$ and $Q$ be a subpath of $P$. Let $u$ and $v$ be the end-vertices of $Q$, we say $Q$ is a $U-V$ path on $P$ if $u \in U$ and $v \in V$. A vertex of a path which is not its end-vertex is an int-vertex of the path. Let $u P v$ denote the $\{u\}-\{v\}$ path on $P$. Futhermore, let $\check{u} P v=u P v-u$, $u P \check{v}=u P v-v$ and $\check{u} \breve{u}=u P v-\{u, v\}$. For a set $\mathcal{P}=\left\{P, P_{1}, P_{2}, \ldots, P_{k-1}\right\} \subseteq \mathcal{L}(G)$ and $i \neq j \in\{1,2, \ldots, k-1\}$, a $V\left(P_{i}\right)-V\left(P_{j}\right)$ path $Q$ on $P$ is $\operatorname{good}$ if $V(Q) \cap V\left(P_{m}\right) \neq \emptyset$ for every $m=1,2, \ldots, k-1$ and neither $P_{i}$ nor $P_{j}$ contain an int-vertex of $Q$. Let $t_{\mathcal{P}}(P)$ be the number of all good paths of $P$ and $t_{\mathcal{P}}^{\prime}(P)$ be the maximum number of all non-intersecting (no edge in common) good paths on $P$. By Proposition 3 in [5], every 2 longest paths intersect. Thus, we have that $t_{\mathcal{P}}(P) \geq t_{\mathcal{P}}^{\prime}(P) \geq 1$ for every $P \in \mathcal{P}$. For a path $P \in \mathcal{P}$, let $X_{\mathcal{P}}^{i}(P)$ denote the set of all vertices of $P$ which are exactly on $i$ paths from $\mathcal{P}$. Let $n_{i}=\left|\bigcup_{P \in \mathcal{P}} X_{\mathcal{P}}^{i}(P)\right|$.

Lemma 5. Let $G$ be a connected graph of order $n$ and $\mathcal{P} \subseteq \mathcal{L}(G)$ with $|\mathcal{P}|=k \geq 3$. If $f(G, \mathcal{P})>0$, then

$$
n \geq \frac{k \cdot \ell(G)+k+(k-2) n_{1}+(k-3) n_{2}+\ldots+n_{k-2}}{k-1} .
$$

Proof. Clearly $n \geq n_{1}+n_{2}+\ldots+n_{k-1}+n_{k}$, where $n_{k}=0$, and $n \geq k(\ell(G)+1)-n_{2}-$ $2 n_{3}-\ldots-(k-3) n_{k-2}-(k-2) n_{k-1}$. Hence $n \geq k \cdot \ell(G)+k-n_{2}-2 n_{3}-\ldots-(k-3) n_{k-2}-$ $(k-2)\left(n-n_{1}-n_{2}-\ldots-n_{k-2}\right)$ and the result follows.

Lemma 6. Let $G$ be a connected graph and $\mathcal{P} \subseteq \mathcal{L}(G)$ with $|\mathcal{P}|=k$. If there exists a path $P \in \mathcal{P}$ with $t_{\mathcal{P}}^{\prime}(P)=1$, then $f(G, \mathcal{P})=0$.

Proof. To the contrary, we suppose there is a path $P=v_{1} v_{2} \ldots v_{\ell(G)+1}$ with $t_{\mathcal{P}}^{\prime}(P)=1$ and $f(G, \mathcal{P})>0$. By $f(G, \mathcal{P})>0$, every good path on $P$ contains an edge. We consider the 'left-most' good path $Q$ on $P$; more formally, we consider the good path $Q=v_{i} v_{i+1} \ldots v_{j}$ such that there is no good path on $P$ containing a vertex $v_{k}$ with $k<i$. Let $\mathcal{P}_{j}$ denote the set of paths of $\mathcal{P}$ which contain $v_{j}$. By the choice of $Q$, some path of $\mathcal{P}_{j}$ contains no vertex $v_{k}$ with $k<j$, and thus the length of $v_{1} v_{2} \ldots v_{j}$ is at most $\frac{1}{2} \ell(G)$. Similarly, we consider the 'right-most' good path $Q^{\prime}=v_{i^{\prime}} v_{i^{\prime}+1} \ldots v_{j^{\prime}}$ and we see that the length of $v_{i^{\prime}} v_{i^{\prime}+1} \ldots v_{\ell(G)+1}$ is at most $\frac{1}{2} \ell(G)$. By the assumption $t_{\mathcal{P}}^{\prime}(P)=1$, the paths $Q$ and $Q^{\prime}$ have an edge in common, so $j>i^{\prime}$, hence the length of $P$ is shorter than $\ell(G)$, a contradiction.

Lemma 7. Let $G$ be a connected graph and $\mathcal{P} \subseteq \mathcal{L}(G)$ with $|\mathcal{P}|=k \geq 3$. Let $P \in \mathcal{P}$ and let $Q$ be a good path on $\mathcal{P}$. Then the following two statements hold:

(i) $f(G, \mathcal{P}) \leq \frac{|V(Q)|-1}{2}(k-1)$;

(ii) $\left|X_{\mathcal{P}}^{1}(P) \cup X_{\mathcal{P}}^{2}(P) \cup \ldots \cup X_{\mathcal{P}}^{k-2}(P)\right| \geq t_{\mathcal{P}}^{\prime}(P)\left(\frac{2}{k-1} f(G, \mathcal{P})-1\right)$.

Proof. Note that if $f(G, \mathcal{P})=0$, then the statement holds. Suppose $f(G, \mathcal{P}) \geq 1$. In particular, every good path on $\mathcal{P}$ contains at least two vertices. Let $x \in V(Q)$ such that 
$\sum_{P^{\prime} \in \mathcal{P}} d_{G}\left(x, P^{\prime}\right) \leq \sum_{P^{\prime} \in \mathcal{P}} d_{G}\left(y, P^{\prime}\right)$ for every $y \in V(Q)$. Then

$$
f(G, \mathcal{P}) \leq \sum_{P^{\prime} \in \mathcal{P}} d_{G}\left(x, P^{\prime}\right) \leq \frac{|V(Q)|-1}{2}(k-1) .
$$

For any path $P$ of $\mathcal{P}$ and any good path $Q^{\prime}$ on $P$, no int-vertex of $Q^{\prime}$ is in $X_{\mathcal{P}}^{k-1}(P)$, therefore $\left|V\left(Q^{\prime}\right) \cap\left(\mid X_{\mathcal{P}}^{1}(P) \cup X_{\mathcal{P}}^{2}(P) \cup \ldots \cup X_{\mathcal{P}}^{k-2}(P)\right)\right| \geq\left|V\left(Q^{\prime}\right)\right|-2 \geq \frac{2}{k-1} f(G, \mathcal{P})-1$. Let $\mathcal{Q}$ be a maximum set of non-intersecting good paths on $P$. By the definition, $t_{\mathcal{P}}^{\prime}(P)=|\mathcal{Q}|$, and we have

$$
\begin{aligned}
\left|X_{\mathcal{P}}^{1}(P) \cup X_{\mathcal{P}}^{2}(P) \cup \ldots \cup X_{\mathcal{P}}^{k-2}(P)\right| \geq\left|\cup_{Q \in \mathcal{Q}}\left(V(Q) \cap\left(X_{\mathcal{P}}^{1}(P) \cup X_{\mathcal{P}}^{2}(P) \cup \ldots \cup X_{\mathcal{P}}^{k-2}(P)\right)\right)\right| \geq \\
\\
\geq \sum_{Q \in \mathcal{Q}}(|V(Q)|-2) \geq t_{\mathcal{P}}^{\prime}(P)\left(\frac{2}{k-1} f(G, \mathcal{P})-1\right) .
\end{aligned}
$$

Corollary 8. Let $G$ be a connected graph and $\mathcal{P} \subseteq \mathcal{L}(G)$ with $|\mathcal{P}|=4$. Let $\mathcal{P}=$ $\left\{P, P_{1}, P_{2}, P_{3}\right\}$ and let $Q$ be a good path on $\mathcal{P}$. Then the following two statements hold:

(i) $f(G, \mathcal{P}) \leq|V(Q)|-1$;

(ii) $\left|X_{\mathcal{P}}^{1}(P) \cup X_{\mathcal{P}}^{2}(P)\right| \geq t_{\mathcal{P}}^{\prime}(P)(f(G, \mathcal{P})-1)$.

Proof. The proof is the same as the proof of Lemma 7 with respect to the following. Let $u, v$ be end-vertices of $Q$. Assume that $Q$ is a $V\left(P_{1}\right)-V\left(P_{2}\right)$ path on $P$ (otherwise we renumber the paths) and we consider a vertex $x \in V(Q) \cap V\left(P_{3}\right)$. Then

$$
f(G, \mathcal{P}) \leq \sum_{P \in \mathcal{P}} d_{G}(x, P)=d_{G}\left(x, P_{1}\right)+d_{G}\left(x, P_{2}\right) \leq d_{G}(u, v) \leq|V(Q)|-1 .
$$

Then we use Corollary 8(i) instead of Lemma 7(i) and the result follows.

Proof of Theorem 因. Suppose that $f(G, \mathcal{P}) \geq 1$. Hence $t_{\mathcal{P}}^{\prime}(P) \geq 2$ by Lemma 6. Let $P \in \mathcal{P}$ be a path minimizing $\left|X_{\mathcal{P}}^{1}(P) \cup X_{\mathcal{P}}^{2}(P) \cup \ldots \cup X_{\mathcal{P}}^{k-2}(P)\right|$. Let $\mathcal{P}-\{P\}=\left\{P_{1}, P_{2}, \ldots, P_{k-1}\right\}$ and $u_{i}, v_{i}$ be the end-vertices of $P_{i}$ for $i \in\{1,2, \ldots, k-1\}$. Assume that $Q$ is a $\operatorname{good} V\left(P_{1}\right)-V\left(P_{2}\right)$ path on $P$ with end-vertices $u, v$ (otherwise we renumber paths $\left.P_{1}, P_{2}, \ldots, P_{k-1}\right)$. Let $R$ be the shortest $\{u\}-V\left(P_{2}\right)$ path on $P_{1}$ and $x \in V(R) \cap V\left(P_{2}\right)$. We may assume that $\left|V\left(u_{2} P_{2} v\right)\right| \leq\left|V\left(u_{2} P_{2} x\right)\right|$ (see Figure $\mathbb{1}$ ).

We have $|V(R)| \geq 2$ from $f(G, \mathcal{P}) \geq 1$ and $|V(Q)| \geq \frac{2 f(G, \mathcal{P})}{k-1}+1$ from Lemma[7(i). Since $v Q \breve{u}$ contains no vertex of $V\left(P_{1}\right), v Q u R x$ is a path in $G$. Futhermore, since $\check{v} Q u P_{1} \check{x}$ contains no vertex of $V\left(P_{2}\right), S_{1}=v_{2} P_{2} v Q u R \check{x}, S_{2}=u_{2} P_{2} v Q u R x P_{2} v_{2}$, and $S_{3}=u_{2} P_{2} x R u Q \check{v}$ are paths in $G$ (see Figure 2).

By comparing the lengths of $P_{2}$ and $S_{1}$ and using Lemma 7 (i) and $|V(R)| \geq 2$, we have

$$
\left|V\left(u_{2} P_{2} v\right)\right|-1 \geq|V(Q)|-1+|V(R)|-2 \geq|V(Q)|-1 \geq \frac{2 f(G, \mathcal{P})}{k-1} .
$$




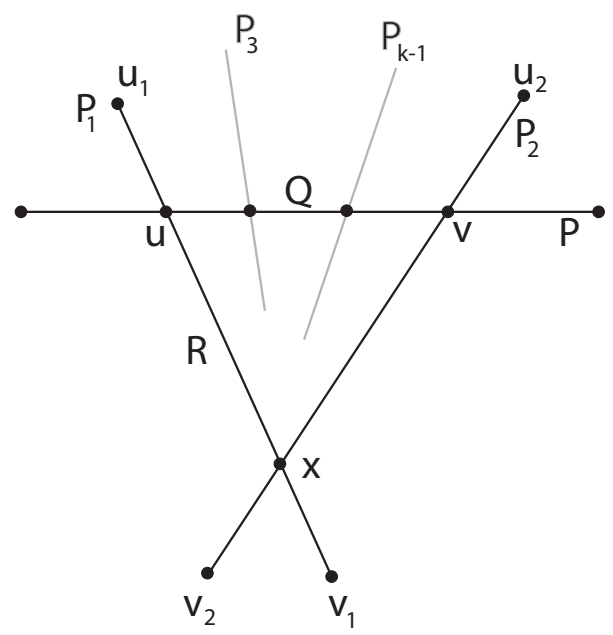

Figure 1: A good $V\left(P_{1}\right)-V\left(P_{2}\right)$ path $Q$ and path $R$

Similarly for $P_{2}$ and $S_{2}$, we have

$$
\left|V\left(v P_{2} x\right)\right|-1 \geq|V(Q)|-1+|V(R)|-1 \geq|V(Q)| \geq \frac{2 f(G, \mathcal{P})}{k-1}+1 .
$$

Also for $P_{2}$ and $S_{3}$, we have

$$
\left|V\left(x P_{2} v_{2}\right)\right|-1 \geq|V(Q)|-1+|V(R)|-2 \geq|V(Q)|-1 \geq \frac{2 f(G, \mathcal{P})}{k-1} .
$$

Therefore all together we have

$$
\begin{gathered}
\ell(G)=\left|V\left(P_{2}\right)\right|-1=\left|V\left(u_{2} P_{2} v\right)\right|-1+\left|V\left(v P_{2} x\right)\right|-1+\left|V\left(x P_{2} v_{2}\right)\right|-1 \geq \\
\geq \frac{2 f(G, \mathcal{P})}{k-1}+\frac{2 f(G, \mathcal{P})}{k-1}+1+\frac{2 f(G, \mathcal{P})}{k-1}=\frac{6 f(G, \mathcal{P})}{k-1}+1 . \quad(*)
\end{gathered}
$$

Clearly $n_{i}=\frac{1}{i} \sum_{P^{\prime} \in \mathcal{P}} X_{\mathcal{P}}^{i}\left(P^{\prime}\right)$. By the choice of $P$ and $t_{\mathcal{P}}^{\prime}\left(P^{\prime}\right) \geq 2$ for every $P^{\prime} \in \mathcal{P}$ together with $(*)$, Lemma 5 , and Lemma 7 we have

$$
\begin{gathered}
n \geq \frac{k \cdot \ell(G)+k+(k-2) \sum_{P^{\prime} \in \mathcal{P}} X_{\mathcal{P}}^{1}\left(P^{\prime}\right)+\frac{k-3}{2} \sum_{P^{\prime} \in \mathcal{P}} X_{\mathcal{P}}^{2}\left(P^{\prime}\right)+\ldots+\frac{1}{k-2} \sum_{P^{\prime} \in \mathcal{P}} X_{\mathcal{P}}^{k-2}\left(P^{\prime}\right)}{k-1} \geq \\
\geq \frac{k \cdot \ell(G)+k+\frac{1}{k-2}\left(\sum_{P^{\prime} \in \mathcal{P}} X_{\mathcal{P}}^{1}\left(P^{\prime}\right)+\sum_{P^{\prime} \in \mathcal{P}} X_{\mathcal{P}}^{2}\left(P^{\prime}\right)+\ldots+\sum_{P^{\prime} \in \mathcal{P}} X_{\mathcal{P}}^{k-2}\left(P^{\prime}\right)\right)}{k-1} \geq \\
\geq \frac{k \cdot \ell(G)+k+\frac{k}{k-2}\left(X_{\mathcal{P}}^{1}(P)+X_{\mathcal{P}}^{2}(P)+\ldots+X_{\mathcal{P}}^{k-2}(P)\right)}{k-1} \geq \\
\geq \frac{k\left(\frac{6 f(G, \mathcal{P})}{k-1}+1\right)+k+\frac{2 k}{k-2}\left(\frac{2}{k-1} f(G, \mathcal{P})-1\right)}{k-1}=\frac{\left(6 k^{2}-8 k\right) f(G, \mathcal{P})+2 k^{3}-8 k^{2}+6 k}{(k-2)(k-1)^{2}},
\end{gathered}
$$



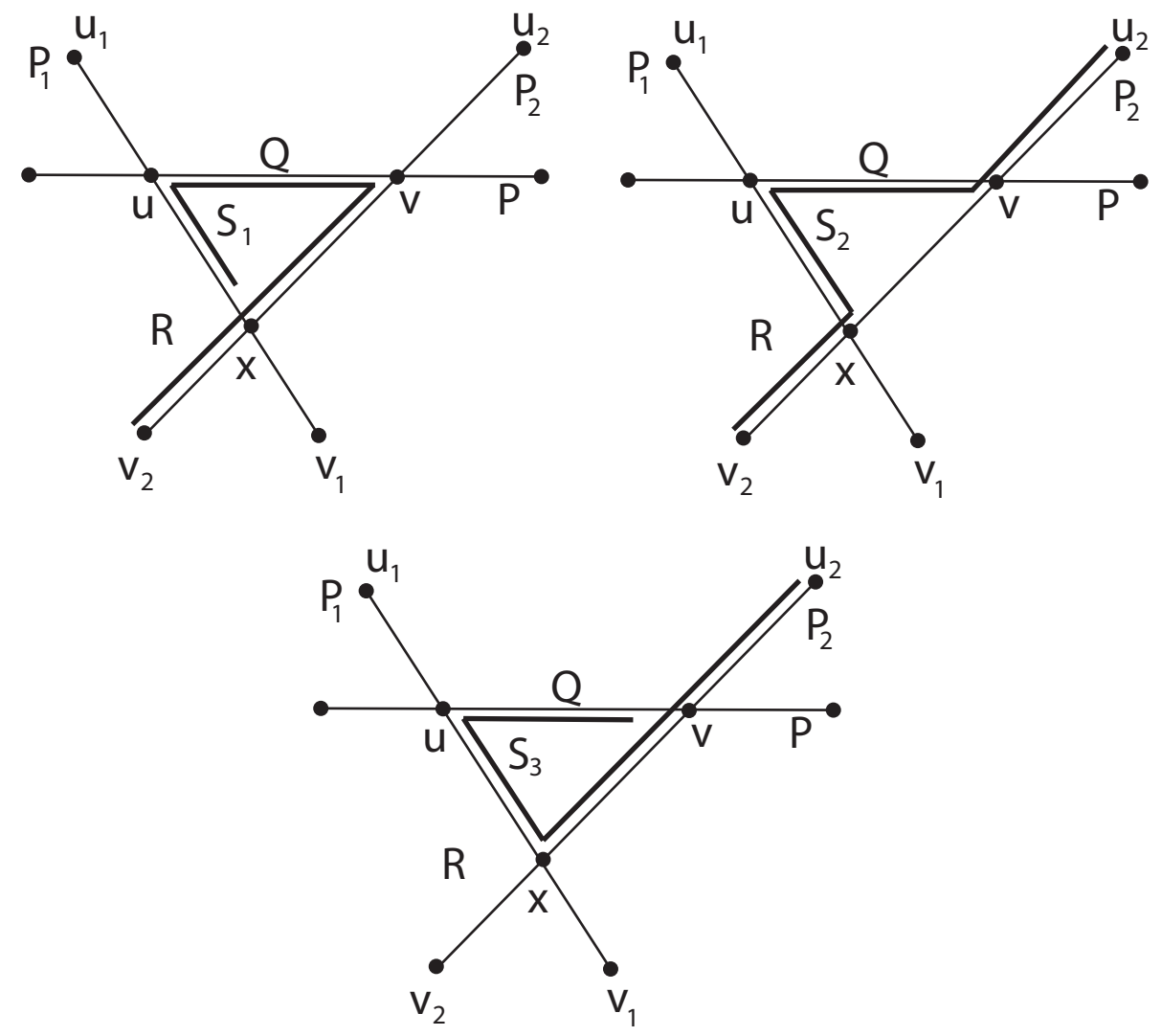

Figure 2: Paths $S_{1}, S_{2}$, and $S_{3}$

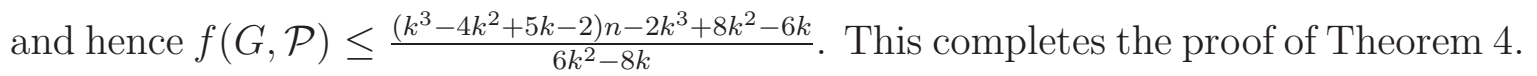

Proof of Theorem 3. We proceed as in the proof of Theorem 4 and use Corollary 8(i) instead of Lemma 7(i). have

By comparing the lengths of $P_{2}$ and $S_{1}$ and using Corollary $8($ i) and $|V(R)| \geq 2$, we

$$
\left|V\left(u_{2} P_{2} v\right)\right|-1 \geq|V(Q)|-1+|V(R)|-2 \geq|V(Q)|-1 \geq f(G, \mathcal{P}) .
$$

Similarly for $S_{2}$ and $S_{3}$, we have

$$
\begin{aligned}
& \left|V\left(v P_{2} x\right)\right|-1 \geq|V(Q)|-1+|V(R)|-1 \geq|V(Q)| \geq f(G, \mathcal{P})+1, \\
& \left|V\left(x P_{2} v_{2}\right)\right|-1 \geq|V(Q)|-1+|V(R)|-2 \geq|V(Q)|-1 \geq f(G, \mathcal{P}) .
\end{aligned}
$$

Therefore all together we have

$$
\begin{aligned}
\ell(G) & =\left|V\left(P_{2}\right)\right|-1=\left|V\left(u_{2} P_{2} v\right)\right|-1+\left|V\left(v P_{2} x\right)\right|-1+\left|V\left(x P_{2} v_{2}\right)\right|-1 \geq \\
& \geq f(G, \mathcal{P})+f(G, \mathcal{P})+1+f(G, \mathcal{P})=3 f(G, \mathcal{P})+1 . \quad(* *)
\end{aligned}
$$


By the choice of $P$ and $t_{\mathcal{P}}^{\prime}\left(P^{\prime}\right) \geq 2$ for every $P^{\prime} \in \mathcal{P}$ together with $(* *)$, Lemma 7 , and Lemma 6 we have

$$
\begin{gathered}
n \geq \frac{4 \ell(G)+4+2 \sum_{P^{\prime} \in \mathcal{P}} X_{\mathcal{P}}^{1}\left(P^{\prime}\right)+\frac{1}{2} \sum_{P^{\prime} \in \mathcal{P}} X_{\mathcal{P}}^{2}\left(P^{\prime}\right)}{3} \geq \\
\geq \frac{4(3 f(G, \mathcal{P})+1)+4+4(f(G, \mathcal{P})-1)}{3}=\frac{16 f(G, \mathcal{P})+4}{3},
\end{gathered}
$$

and hence $f(G, \mathcal{P}) \leq \frac{3 n-4}{16}$. This completes the proof of Theorem 3 .

\section{Conclusion}

As it was mentioned in Introduction, we extend Conjecture 1 to Conjecture 9.

Conjecture 9. For every connected graph, any $k$ of its longest paths have a common vertex for $3 \leq k \leq 6$.

Conjecture 10 is an extension of a Conjecture stated in [5] for 3 longest paths. We prove that Conjecture 10 is equivalent with Conjecture 9.

Conjecture 10. There exists a sublinear function $g$ such that for every connected graph $G$ of order $n$ and every subset $\mathcal{P}$ of $\mathcal{L}(G)$ with $3 \leq|\mathcal{P}| \leq 6, f(G, \mathcal{P}) \leq g(n)$.

Let $\mathcal{G}_{n}$ be a class of connected graphs of order at least $n$. In other words, using $d_{k}\left(\mathcal{G}_{n}\right)$ with $3 \leq k \leq 6$, Conjecture 10 translates into the following statement. The path distance ratio $d_{k}\left(\mathcal{G}_{n}\right)$ goes to 0 as $n$ goes to infinity.

Theorem 11. Conjecture 9 is true if and only if Conjecture 10 is true.

Proof. Suppose Conjecture 9 holds. For every set $\mathcal{P}$ of $k$ longest paths $(3 \leq k \leq 6)$ of every connected graph $G$, we have $f(G, \mathcal{P})=0$. Thus any non-negative sublinear function implies that Conjecture 10 holds.

Suppose Conjecture 10 holds. We prove the contrapositive statement, that is, if Conjecture 9 is not true, then neither is Conjecture 10, For $3 \leq k \leq 6$, we consider a connected graph $G$ and a set $\mathcal{P}$ of its $k$ longest paths so that they have no common vertex. We extend $G$ by adding a pendant edge to every vertex, which is an end-vertex of a path of $\mathcal{P}$, and we note that each path of $\mathcal{P}$ prolonged with two of these new edges is a longest path in the extended graph. For a non-negative integer $t$, we subdivide every edge of the extended graph $t$ times and we observe that the corresponding $k$ paths, say $\mathcal{P}_{t}$, are longest paths in the resulting graph $G_{t}$. Let $n$ be the number of vertices and $m$ the number of edges of $G$. We see that $G_{t}$ has at most $n+t(m+2 k)$ vertices. By construction, $f\left(G_{t}, \mathcal{P}_{t}\right) \geq t$. We consider the sequence of graphs $\left(G_{t}\right)_{t=1}^{\infty}$ and we note that $f\left(G_{t}, \mathcal{P}_{t}\right)$ cannot be bounded from above by a sublinear function. 


\section{Acknowledgements}

This work was partly supported by the project LO1506 of the Czech Ministry of Education, Youth and Sports.

The first and third authors were supported by project GA14-19503S of the Grant Agency of the Czech Republic.

The second author's research is supported by Grant-in-Aid for Scientic Research (C) (15K04979). Also, this work was partially completed during a visit of the second author

to the University of West Bohemia. He wishes to express his thanks for the generous hospitality.

\section{References}

[1] M. Axenovich, When do 3 longest paths have a common vertex? Discrete Math. Alg. Appl. 1 (2009) 115-120.

[2] P. Balister, E. Györi, J. Lehel, R. Schelp, Longest paths in circular arc graphs, Combin. Probab. Comput. 13 (2004) 311-317.

[3] S. F. de Rezende, C. G. Fernandes, D. M. Martin, Y. Wakabayashi, Intersecting longest paths, Discrete Math. 313 (2013) 1401-1408.

[4] P. Erdös, G. Katona (Eds.), Theory of Graphs, Proceedings of the Colloquium Hald at Tihany, Hungary, 1966, Academic Press, New York, 1968, Problem 4 (T. Gallai), p.362.

[5] S. Fujita, M. Furuya, R. Naserasr, K. Ozeki, A New Approach Towards a Conjecture on Intersecting Three Longest Paths, arXiv:1503.01219v2, 2015.

[6] S. Klavžar, M. Petkovšek, Graphs with nonempty intersection of longest paths, Ars Combin. 26 (1990) 43-52.

[7] Z. Skupień, Smallest sets of longest paths with empty intersection. Combin. Probab. Comput. 5 (1996), no. 4, 429-436.

[8] C. Thomassen, Planar and infinite hypohamiltonian and hypotraceable graphs, Discrete Math. 14 (1976) 377-389.

[9] H. Walther, Über die Nichtexistenz eines Knotenpunktes durch den allen längsten Wege eines Graphen gehen, J. Combin. Theory 6 (1969) 1-6.

[10] H. Walther, H. J. Voss, Über Kreise in Graphen, VED Deutcher Verlag der Wissenschaften, 1974.

[11] T. Zamfirescu, On longest path and circuits in graphs, Math. Scand. 38 (1976) 211-239. 
[12] T. Zamfirescu, Intersecting longest path or cycles: short survey, An. Univ. Craiova Ser. Mat. Inform. 28 (2001) 1-9. 